\title{
Depth-sensitive characterization of surface magnetic properties of as-quenched FeNbB ribbons
}

\author{
O. Životský, K. Postava, K. Hrabovská, A. Hendrych, J. Pištora \\ Department of Physics, Technical University of Ostrava, 17.listopadu 15, 70833 \\ Ostrava-Poruba, Czech Republic
}

\section{Kraus}

Institute of Physics, Academy of Sciences of the Czech Republic, Na Slovance 2, 18221 Prague 8, Czech Republic

The longitudinal magneto-optical Kerr effect (MOKE) is used to study the surface magnetic properties of as-quenched FeNbB ribbons. MOKE surface hysteresis loops measured from both ribbon sides confirm different magnetic behavior. Wheel ribbon side shows heterogeneous (crystalline/amorphous) properties, thickness of crystalline phase (about $3 \mathrm{~nm}$ ) was established by comparing the measured magneto-optical angles of Kerr rotation and ellipticity at different incident angles with the theoretical model. Effective crystalline phase observed at shiny ribbon side is harder (coercive field about 40 Oe) than that on wheel side and penetrates deeper into the material volume. Its thickness $1.3 \mu \mathrm{m}$ estimated from the weight reduction of the ribbon during surface etching is in good agreement with cross-section image obtained using the scanning electron microscopy (SEM). The sources of magnetoelastic anisotropy were identified in the bulk as well as on the ribbon surface using the magneto-optical Kerr microscopy.

Key words: Amorphous and nanocrystalline magnetic materials, Magnetooptic 
Kerr effect, Scanning electron microscopy, Depth profile

PACS: 75.30.Kz, 75.50.Bb, 75.70.-i, 75.70.Rf, 75.75.+a, 76.80.+y

\section{INTRODUCTION}

Amorphous and nanocrystalline Fe-based ribbons are systematically studied for many years due to excellent soft magnetic properties. Their progress and applicability has rapidly grown in seventies of 20th century, when the commercial production of the amorphous alloys called the metallic glasses (Metglass) began. Especially the Metglass alloys with the compositions Fe-B and Fe-BSi exhibited much lower coercive force and higher magnetic permeability in comparison to other soft magnetic materials [1-4]. Further development and improvement of magnetic softness and thermal stability was discovered at materials with heterogeneous (crystalline / amorphous) structure often called nanocrystalline soft magnetic materials. The best known commercial alloys are NANOPERM, FINEMET, and HITPERM [5,6]. As a consequence the nanocrystalline soft magnetic materials showed great potentials in many industrial applications like the transformer cores, the switched-mode power supplies, the ground fault interrupters or the magnetic sensors [7-9].

Present technological trends in investigations of nanocrystalline materials are mainly focused on the improvement of their magnetic and mechanical properties, decreasing the size of crystalline grains, controling the volume ratio between the crystalline and amorphous phases, eliminating the surface corrosions, and the surface magnetic domains observations that correspond to the anisotropy distribution. Mentioned properties can be achieved in ribbon 
as-quenched state as well as by sufficient postpreparation treatment like the annealing at sufficient temperatures, annealing in applied magnetic field or by stress applications [10-13]. Moreover, there are introduced new material compositions originating by adding new elements ( $\mathrm{Ni}, \mathrm{Mn}, \mathrm{Mo})$ to the traditional Fe-based nanocrystalline alloys [14-16]. Hence there is a need to use advanced surface and bulk sensitive methods and investigate the depth profile of the magnetic properties in the near-surface region of these alloys.

Recently we investigated the surface and bulk magnetic properties of asquenched FeNbB ribbons prepared by planar flow casting [17]. Main emphasis was devoted to the surface sensitive methods like the magneto-optical Kerr effect (MOKE) and the conversion electron Mössbauer spectroscopy (CEMS). Due to lower searching depths of both methods (MOKE - $30 \mathrm{~nm}$, CEMS 200-300 nm) [18] we detected the contribution of thin crystalline phase close to the ribbon wheel side, i.e. the side in direct contact with quenching wheel. Xray diffraction (XRD) measurements shown that crystallites are highly $\left(\begin{array}{lll}2 & 0 & 0\end{array}\right)$ textured. Moreover, after $10 \mathrm{~s}$ of etching in diluted $\mathrm{HNO}_{3}$, which corresponded to removing about $50 \mathrm{~nm}$ from the ribbon wheel side, the sharp $\alpha$-Fe diffraction peak disappeared and only broad amorphous peaks were observed. Consequently the influence of the annealing on the crystalline phase and ribbon anisotropy was studied in Ref. [19].

In this paper we continue in our studies on the as-quenched FeNbB ribbons and our attention is focused on three main problems. Firstly, we try to estimate more precisely the thickness of crystalline phase from the ribbon wheel side. To solve this problem we use the surface-sensitive method based on the measurement of complete complex longitudinal Kerr effect at variable incident angle [19]. Secondly, we investigate in details the shiny ribbon side, i.e. the 
side in contact with the air during preparation process. It seems that thicker crystalline phase observed from the shiny side by the XRD and the CEMS methods is exchange-biased with the soft amorphous bulk [20]. Hence the study of the thickness and magnetic properties of such layer is important for understanding the bias phenomenon in these materials. Finally we deal with the anisotropy distribution on the ribbon surface using the magneto-optical Kerr microscopy.

\section{EXPERIMENTAL}

$10 \mathrm{~mm}$ wide and $28 \mu \mathrm{m}$ thick as-quenched $\mathrm{Fe}_{80.5} \mathrm{Nb}_{6.9} \mathrm{~B}_{12.6}$ ribbons were prepared by conventional planar method and additionally spark-cut to the $9 \mathrm{~mm}$ discs to eliminate the in-plane shape anisotropy. MOKE experimental setup based on differential intensity method consists of the semiconductor laser working at wavelength of $670 \mathrm{~nm}$, the polarizer, the quarter-wave plate used as retarder, the Wollaston prism, and two photodiodes [19]. Approximately circular laser spot about $0.5 \mathrm{~mm}$ in diameter was focused on the center of the circular sample. The magneto-optical angles of Kerr rotation $\theta_{K s, p}$ without the retarder and Kerr ellipticity $\epsilon_{K s, p}$ using the quarter-wave plate for $s$ - and $p$-polarized light are measured at different incident angles. The longitudinal hysteresis loops detecting the magnetization component in the sample plane and in the plane of incidence are obtained [21]. We did not observe any polar (out of plane) component of magnetization. All measurements were done at room temperature and in an open air.

SEM digital images of the sample cross-section were obtained from both ribbon sides by PHILIPS XL-30 scanning electron microscope. Images were taken at 
$25 \mathrm{kV}, 2000 \times$ magnification using the secondary electrons. Dispersion X-ray spectrometer EDAX implemented into the microscope enables to characterize the average chemical composition of detected phases.

Zeiss optical microscope specially designed for MO Kerr microscopy is used to magnetic domain observations. Light emitted from the arc xenon lamp is focused on the plane of the aperture diaphragm that determines which part of the sample is illuminated and also specifies the incident angle. Two main configurations are distinguished [22]. The off-centered diaphragm results in the oblique incident angle and therefore such configuration is sensitive to longitudinal MOKE. However, at oblique incidence angle, the mixing of longitudinal and polar magnetization components is always observed. Conversely centered diaphragm leads to the perpendicular incidence and to the sensitivity to sole polar MOKE. Polarized light is focused on the sample surface by the polarization objective and after reflection transmitted throught the analyzer almost crossed with the polarizer. Resulting image of the domain patterns observed using the high resolution CCD camera is obtained as difference of an image in magnetically saturated state and a picture containing magnetic contrast. The observations were made only from the sufficiently smooth shiny side of the ribbon. The wheel side requires special surface preparation like polishing or etching, and this process completely destroys the informations from the very thin crystalline layer on the wheel side.

\section{RESULTS AND DISCUSSIONS}

Figures 1a, b and 1c, d show the magneto-optical Kerr angles of $\theta_{K s}$ and $\epsilon_{K s}$ from the wheel and shiny ribbon side at the incident angle of $80^{\circ}$. Ex- 
ternal magnetic field $H$ is applied along the original ribbon axis. The shape differences in the case of wheel and shiny loops confirm different magnetic properties of the two ribbon sides with the coercive fields $\left(H_{c}\right) 10$ Oe and 40 Oe, respectively.

\subsection{Wheel ribbon side}

In general the surface sensitive MOKE methods are possible to distinguish the contributions of two or more phases in different depths of investigated materials. In such case the measured hysteresis loop is composed by adding the loops from partial phases [23]. Moreover the shape of the loop changes by tuning the parameters like the polarization of incident light ( $s$ or $p$ ), the Kerr angle (rotation or ellipticity) [23], the light wavelength [24], or the angle of incidence.

Similar behavior is clearly shown at loops from wheel side of FeNbB ribbon (Figs. 1a, b). Different shapes for $\theta_{K s}, \epsilon_{K s}$ quantities and also for various incident angles (in Ref. [17] the loops are shown at $60^{\circ}$ ) confirm that the nearsurface region is inhomogeneous and MOKE detects the contributions of two phases in different depths, crystalline and amorphous ones. As we already mentioned, partial surface nanocrystallization with strong texture was confirmed also by XRD measurements and the CEMS, which determine $10 \%$ of crystalline phase in region of thickness of about 200-300 nm [17]. Supplemented measurements using the transmission electron microscopy (TEM) show the $\alpha$-Fe crystallites with average size of grains about $100 \mathrm{~nm}$ situated near the wheel surface. 
Existence and thickness of crystalline phase in the thin surface layer was checked in two ways: i) by electrochemical surface polishing ii) by etching of surface in diluted $\mathrm{HNO}_{3}$. After short polishing and etching, the surface crystalline layer was removed, as confirmed by XRD. The measured MOKE angles are also substantially changed. The shapes of magneto-optical hysteresis loops became the same for both polarizations and the Kerr angles and the coercive field decreased to 3 Oe. These conclusions enabled us to sketch the schematic model of the near-surface region from wheel side, see lower part of Figure 2.

Optical and magneto-optical parameters of remaining amorphous phase can be established by comparing the data measured in the range of incident angles from $20^{\circ}$ to $80^{\circ}$, with the theoretical model based on the light propagation in layered anisotropic media [25]. Figures $3 \mathrm{a}$ and $3 \mathrm{~b}$ show the angular dependence of the calculated (solid and dashed lines) and measured MO angles (circles and squares) in the case of electrochemically polished surface. Refractive index $n_{a}$ and Voight constant $Q_{a}$ of amorphous phase were calculated as: $n_{a}=$ $3.02-i 4.18 ; Q_{a}=0.0156+i 0.0025$.

On the other hand, etching of the surface caused the chemical reaction of $\mathrm{HNO}_{3}$ with the ribbon and led to the origin of surface oxide layer with the refraction index $n_{o}$ and thickness $d_{o}$. We found out that the thickness of oxide layer changes with etching times. We also independently confirmed by CEMS that it does not contain iron oxides. In subplots c and d of Fig. 3, the measured data, in the case of 10 s etching, are compared with the theoretical model. Note that the presence of oxide layer causes the shift of the maxima of the magnetooptical angles towards lower incidence angles. We took the fitted $n_{a}, Q_{a}$ as constants and calculated the parameters $n_{o}=1.88$ and $d_{o}=20 \mathrm{~nm}$ of oxide 
layer.

Knowing the MO parameters of amorphous phase we can now analyze MOKE of the original as-quenched ribbon. We assume that the near-surface region of AQ sample consists of an upper hard crystalline layer and the soft amorphous bulk (see Fig. 2) with the parameters established in the previous step. To complete the ribbon depth profile from the wheel side we fitted all data measured on AQ ribbon with the two-layer model (subplots 3e, 3f) and found the parameters of the crystalline phase $n_{c}=2.92-i 3.07 ; Q_{c}=0.0236+i 0.0024$ with the thickness $d_{c}=3 \mathrm{~nm}$. The refractive index $n_{c}$ is very close to the pure iron $\left(n_{F e}=2.90-i 3.07\right)[26]$, which confirms the CEMS and XRD results.

\subsection{Shiny ribbon side}

We can see that the shapes of $\theta_{K s}$ and $\epsilon_{K s}$ hysteresis loops (subplots 1c, d) measured on the shiny side are the same. In contrast to the wheel side this behavior can be explained by a single-layer model (see Fig. 2) for an effective optical medium composed of the crystallites more or less randomly oriented in the amorphous phase with the thickness, which is larger than the penetration depth of light $(30 \mathrm{~nm})$. Higher value of coercive field indicates that the surface effective crystalline layer is harder than that on the wheel side. In accordance with MOKE measurements, the existence of crystalline layer from shiny side was confirmed also by CEMS and XRD. CEMS measurements showed only $5 \%$ of the crystalline $\alpha$-Fe phase in the near-surface region of shiny side, while the remainder was amorphous [17]. Calculated effective optical and magnetooptical parameters $\left(n_{S}=2.87-i 3.46 ; Q_{S}=0.0196+i 0.0044\right)$ of detected layer can be also compared to pure iron [26]. 
Depth profile from the shiny side was investigated using the measurements, when the ribbon surface was gradually etched in 1:2 water solution of diluted $\mathrm{HNO}_{3}$. Figure 4 shows the measured hysteresis loops of the Kerr rotation (Fig. 4a) and the Kerr ellipticity (Fig. 4b) at various etching times $t_{\text {etch. The }}$ loops shown in Fig. 4 were obtained for $s$-polarized light at the incidence angle of $45^{\circ}$. While during the first $30 \mathrm{~s}$ of etching no changes of shapes and coercive fields of MOKE hysteresis loops are seen, different magnetic behavior is observed for higher etching times. The increase of $H_{c}$ and $H_{s}$ (saturation field) for etching times $t_{\text {etch }} \geq 35 \mathrm{~s}$ indicates that surface effective layer becomes magnetically harder and the volume ratio of crystalline to amorphous phase changes. Based on these results we expect the gradient distribution of crystallites in the layer.

After $80 \mathrm{~s}$ of etching (circle line in Fig. 4), the harder effective layer is completely removed and only the magnetooptic contribution from the amorphous bulk is observed. The hysteresis loops for both MO angles are almost rectangular with the coercive field of 10 Oe. The bulk $H_{c}$ obtained from the shiny side is larger than that from the wheel side $\left(H_{c}=3 \mathrm{Oe}\right)$. The thickness of the surface effective layer can be estimated by measuring the sample weight before and after etching. We found out that $80 \mathrm{~s}$ of etching in 1:2 diluted $\mathrm{HNO}_{3}$ removes about $9.6 \%$ of the ribbon weight. Because we etched both sides of the ribbon, the thickness of the effective layer on the shiny side can be estimated to about $1.3 \mu \mathrm{m}$.

This fact was proved by observing the ribbon cross-section in the scanning electron microscope with the magnification of 2000. For this experiment $1 \mathrm{~cm}$ long ribbon was fixed in vacuum apparatus into an epoxide resin, then refined and polished. Figure 5a shows a sharp phase interface between the crystalline 
effective layer (denoted by white colour in the picture) and the amorphous bulk (grey colour in Fig. 5a). Upper part of Fig. 5a represents the epoxide resin, in which the sample was embedded. From the cross-section it can be established that surface effective layer is roughly $1.5 \mu \mathrm{m}$, what is in good agreement with MOKE experiments. Note that thickness of this layer is not uniform along the whole ribbon length.

Energy dispersive X-ray spectrometry (EDS) is an analytical technique used for the elemental analysis and chemical characterization of a sample. EDS detector measures relative counts of detected x-rays emitted due to interaction between the electron beam from the SEM and the sample surface. Phases as small as $1 \mu \mathrm{m}$ can be analyzed using this technique. Figure $5 \mathrm{~b}$ shows the EDS spectrum of effective crystalline phase in the spectral range up to $8 \mathrm{keV}$. As we expected it consists mainly of the $\mathrm{Fe}$ and $\mathrm{Nb}$ elements. However the oxygen, the carbon, and the aluminium elements observed around the energies $0.52 \mathrm{keV}, 0.25 \mathrm{keV}$, and $1.5 \mathrm{keV}$ were embedded into the layer during the polishing of the sample casted in the resin $\left(\mathrm{Al}_{2} \mathrm{O}_{3}\right.$-based polishing suspension was used). In comparison to bulk phase the effective crystalline phase exhibits higher amount of the FeKa element (approximately 1.09 times) and the NbLa element (2 times).

Magnetic anisotropy is one of the most important properties for practical applicability of such materials that closely relates with the arrangement of domains and domain walls in the amorphous bulk and on the ribbon surface. As we expected the magnetic microstructure of as-quenched FeNbB ribbon can be classified as disorder. Figure 6 shows the magnetic domain patterns observed in the amorphous bulk phase. We used the longitudinal configuration with the off-centered aperture iris, as shown in left subplot. Both domains types, wide 
$180^{\circ}$-walls domains with the widths up to $100 \mu \mathrm{m}$ and narrow fingerprint-like (maze) domains, are the consequence of the internal stresses originating during planar flow casting process. However, the magnetization is connected to the stress by the magnetostriction constant $\lambda_{s}$ that is usually not zero in the NANOPERM-type alloys. In our case the value of $\lambda_{s}$ in amorphous bulk was estimated to $10.8 \times 10^{-6}$. Note that similar stress-dominating domain patterns were presented in many other Fe-based alloys with the positive magnetostriction $[27,28]$.

Separation of the in-plane and out-of-plane magnetization components was carried out by focusing the light on the center of the aperture diaphragm. Because the wide-curved domains completely disappeared in this configuration, we expect that they originate in the areas with dominating tensile stress and their direction follows the magnetoelastic in-plane easy axis everythere on the surface. As can be seen the local in-plane easy axis in Fig. 6 is inclined approximately $45^{\circ}$ from the ribbon axis. On the other hand, the fingerprint domains exhibit the polar magnetization component in the ribbon volume, where the compressive stress dominates. In conclusion we must emphasize that both domain types are visible also on the surface of the effective crystalline layer. It means that partial surface crystallization did not cause the ordered magnetic state, and the domain dimensions are similar like in the bulk phase.

\section{CONCLUSIONS}

Surface magnetic properties and depth profile of as-quenched FeNbB ribbons were investigated. Both sides of the ribbon exhibit the presence of crystalline phase on the surface, which is exchange-biased with the amorphous remainder. 
We were mainly oriented on the effective crystalline layer observed from shiny side, because its exchange bias effects are approximately four times stronger than on the wheel side. Magnetic properties, anisotropy, thickness, and chemical composition were analyzed using the MOKE, the SEM, and the EDS methods.

\section{Acknowledgments}

This work has been partially supported by the grants KAN 400100653, MSM6198910016, and also from the Czech Grant Agency (202/06/0531, 102/08/0743).

\section{References}

[1] F.E. Luborsky and J.L. Walter, IEEE Trans. Magn. MAG-13 (1977) 1635.

[2] H.R. Hilzinger, A. Mager, and H. Warlimont, J. Magn. Magn. Mater. 9 (1978) 191.

[3] Y.D. Yao and S.T. Lin, Chinese Journal of Physics 18 (1980) 43.

[4] I. Vincze, D.S. Boudreaux, M. Tegze, Phys. Rev. B19 (1979) 4896.

[5] M.E. McHenry, F. Johnson, H. Okumura, T. Ohkubo, V.R.V. Ramanan, D.E. Laughlin, Scripta Materialia 48 (2003) 881.

[6] T. Kulik, J. Ferenc, and M. Kowalczyk, Journal of Materials Processing Technology 162 (2005) 215.

[7] G. Herzer, Scripta Metallurgica et Materialia. 33 (1995) 1741.

[8] T. Naohara, Appl. Phys. Lett. 68 (1996) 1012. 
[9] K. Suzuki, A. Makino, A. Inoue, and T. Masumoto, J. Appl. Phys. 74 (1993) 3316.

[10] N. Ito and K. Suzuki, J. Appl. Phys. 97 (2005) 10F503.

[11] A. Hirata, Y. Hirotsu, E. Matsubara, T. Ohkubo, and K. Hono, Phys. Rev. B74 (2006) 184204.

[12] P. Vavassori, L. Callegaro, E. Puppin, F. Malizia, F. Ronconi, J. Magn. Magn. Mater. 157-158 (1996) 171.

[13] W. Lu, B. Yan, and R. Tang, Journal of Alloys and Compounds 425 (2006) 406.

[14] L. Fernández et al., J. Non-Cryst. Sol. 353 (2007) 777.

[15] C. Gomez-Polo, J.I. Perez-Landazabal, V. Recarte, P. Mendoza Zelis, Y.F. Li, M. Vazquez, J. Magn. Magn. Mater. 290-291 (2005) 1517.

[16] J. Čermák and I. Stloukal, Journal of Physics and Chemistry of Solids 68 (2007) 1249.

[17] O. Životský et al., J. Magn. Magn. Mater. 320 (2008) 1535.

[18] J. Juraszek, O. Zivotsky, H. Chiron, C. Vaudolon, and J. Teillet, Rev. Sci. Inst., to be published.

[19] O. Životský, K. Postava, L. Kraus, K. Hrabovská, and J. Pištora, Proc of SPIE 6585 (2007) 65851P.

[20] L. Kraus, O. Životský, K. Postava, P. Švec, and D. Janičkovič, IEEE Trans. Magn. (2008) in press.

[21] O. Životský, L. Kraus, K. Postava, M. Foldyna, J. Pištora, J. Appl. Phys. 99 (2006) 08F107.

[22] R. Schäfer, Investigation of Domains and Dynamics of Domain Walls by the Magneto-optical Kerr-effect, in: Handbook of Magnetism and Advanced Magnetic Materials, John Willey \& Sons, 2007. 
[23] M. Nývlt, J. Ferré, J. P. Jamet, P. Houdy, P. Boher, Š. Višňovský, R. Urban, and R. Lopušník, J. Magn. Magn. Mater. 156 (1996) 175.

[24] G. Pénissard, P. Meyer, J. Ferré, and D. Renard, J. Magn. Magn. Mater. 146 (1995) 55.

[25] Š. Višňovský, Optics in Magnetic Multilayers and Nanostructures, CRC Press, Taylor\&Francis, 2006.

[26] P. B. Johnson and R. W. Christy, Phys. Rev. B9 (1974) 5056.

[27] M. Tejedor and B. Hernando, J. Phys. D: Appl. Phys. 13 (1980) 1709.

[28] A. Hubert, R. Schäfer, Magnetic Domains, Springer, New York, 2000, p. 436. 
Fig. 1. Surface magneto-optical hysteresis loops obtained on $9 \mathrm{~mm}$ disc of as-quenched FeNbB ribbon at the inspected wavelength of $670 \mathrm{~nm}$. Subplots a, $\mathrm{b}$ and $\mathrm{c}, \mathrm{d}$ correspond to the magneto-optical quantities $\theta_{K s}$ and $\epsilon_{K s}$ measured from wheel and shiny ribbon side.

Fig. 2. Schematic model suggesting the cross-section of AQ FeNbB ribbon. $n_{i}$ and $Q_{i}$, where $i=a, c, S$ describe the refractive indices and the Voight constants of amorphous, crystalline, and effective crystalline phase.

Fig. 3. Wheel side models describing the dependence of the Kerr magneto-optical angles on the incidence angle. Subplots a, b and c, d correspond to the experiments with wheel side polishing and etching, when crystalline phase is removed. Thickness and magneto-optical parameters of crystalline phase in the case of non-polished and non-etched ribbon were obtained by comparing the measured data with two-layer model (subplots e, f).

Fig. 4. Magneto-optical hysteresis loops obtained from the shiny ribbon side at different etching times $t_{\text {etch }}$. Left and right subplots correspond to the $\theta_{K s}$ and $\epsilon_{K s}$ MO angles.

Fig. 5. a) SEM image mapping the cross-section of the shiny ribbon side; b) EDS spectrum showing the elements composition of effective crystalline layer.

Fig. 6. Zero-field magnetic domains measured in the bulk of as-quenched FeNbB ribbon. The wide-curved domains lie in the plane of the ribbon, while the fingerprint domains with maximal width up to $3 \mu \mathrm{m}$ indicate the presence of out-of-plane magnetization component. 

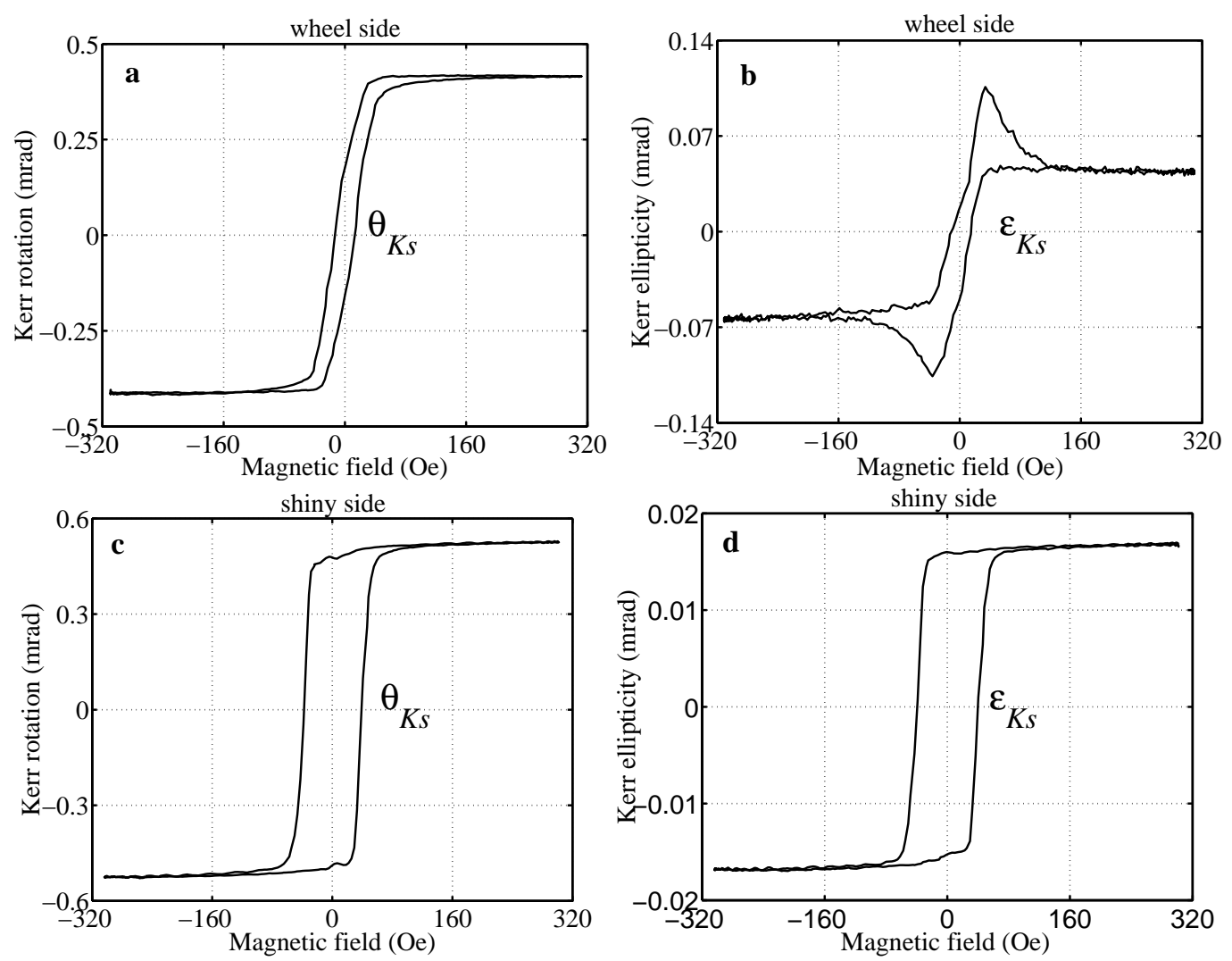

Fig. 1 


\section{shiny side}

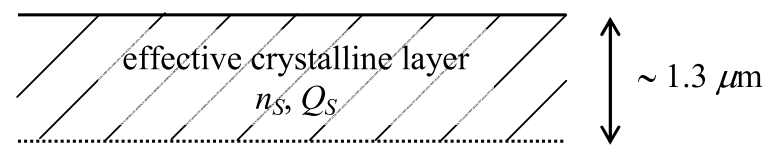

amorphous bulk

$n_{a}, Q_{a}$

$\frac{\text { crystailine layer }-n_{c}, Q_{c} \cdots \cdots \cdots \cdots \cdots \cdots \cdots \cdots \cdots \cdots}{\text { wheel side }} \uparrow^{\downarrow} \sim 3 \mathrm{~nm}$

Fig. 2 

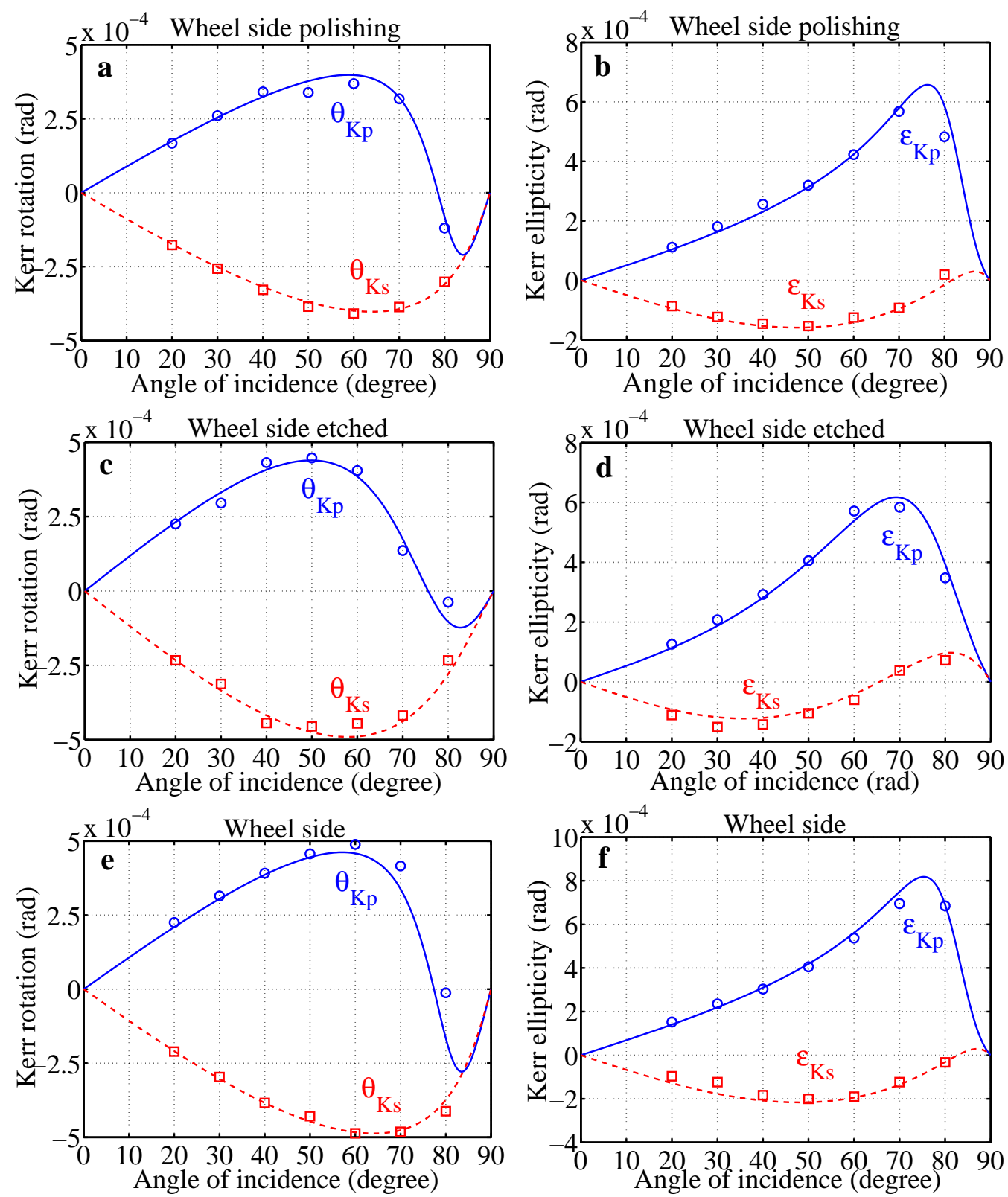

Fig. 3 

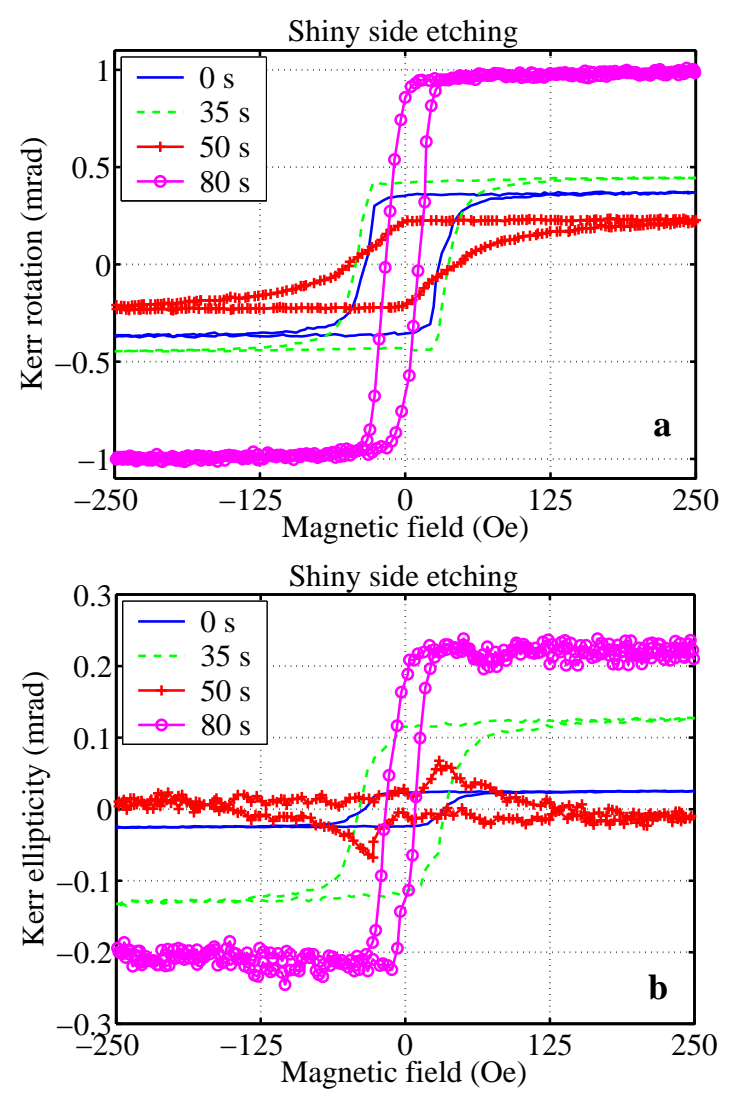

Fig. 4 


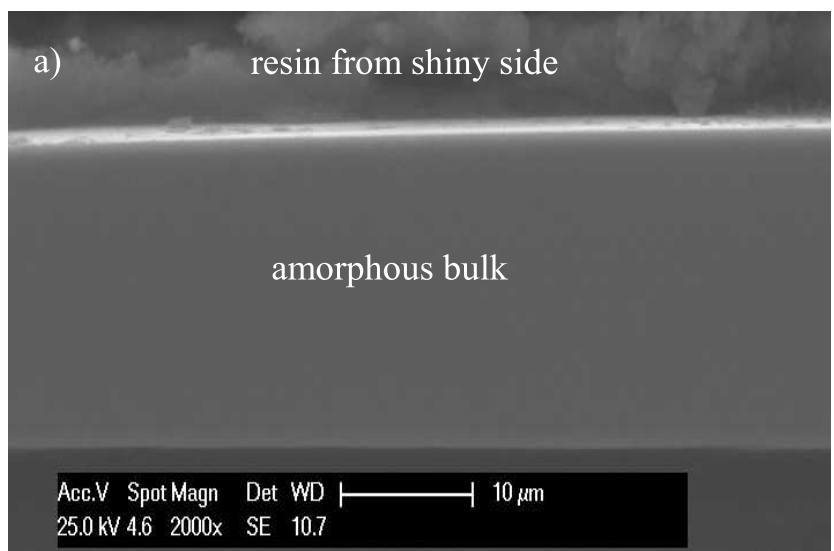

b) effective crystalline layer

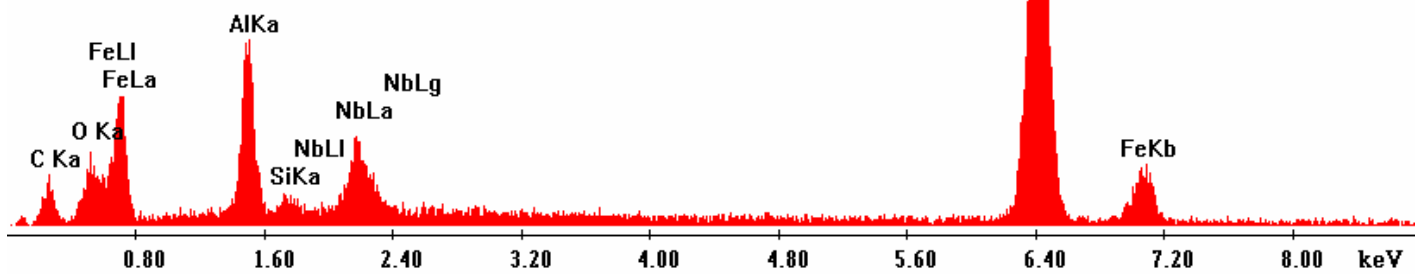

Fig. 5 


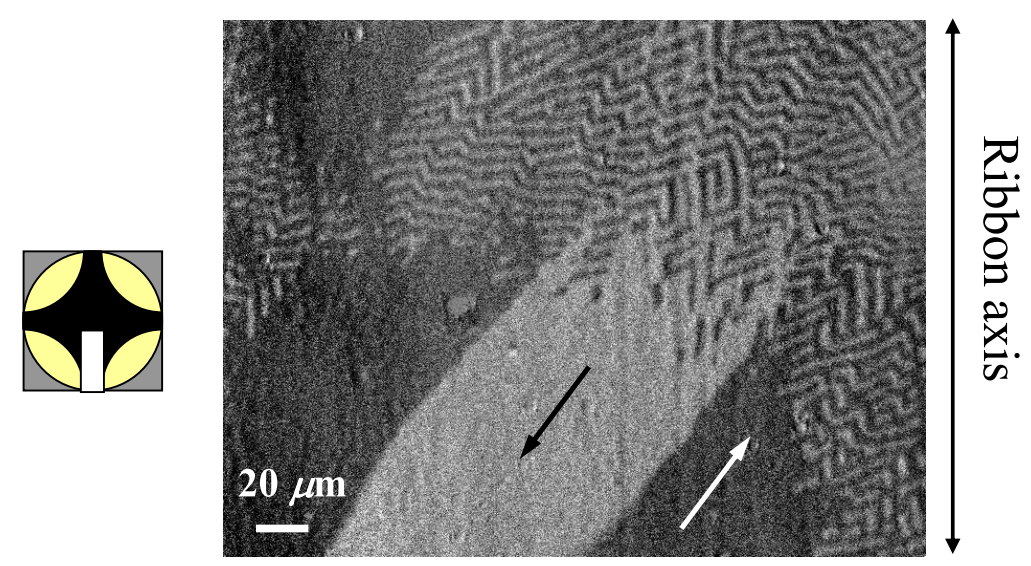

Fig. 6 